\title{
Investigating Bullying Determinants and Typologies with Social Network Analysis
}

\author{
Alexandra Bekiari, Vasiliki Pachi, Nikolaos Hasanagas \\ Faculty of Physical Education and Sports Sciences, University of Thessaly, Trikala, Greece \\ Email:sandrab@pe.uth.gr
}

How to cite this paper: Bekiari, A., Pachi, V. and Hasanagas, N. (2017) Investigating Bullying Determinants and Typologies with Social Network Analysis. Journal of Computer and Communications, 5, 11-27. https://doi.org/10.4236/jcc.2017.57002

Received: April 3, 2017

Accepted: May 8, 2017

Published: May 11, 2017

Copyright $\odot 2017$ by authors and Scientific Research Publishing Inc. This work is licensed under the Creative Commons Attribution International License (CC BY 4.0).

http://creativecommons.org/licenses/by/4.0/ (c) (i) Open Access

\begin{abstract}
Aim of this paper is to detect determinants and to suggest a typology bullying. Four network samples of 218 students in total $($ male $=92$, female $=126)$ at the Physical Education and Sport Sciences Dept. and the Veterinary Dept. of the University of Thessaly have been collected in 2017. Standardized questionnaire composed of network and non-network part was answered. Social network analysis and cross-sectional statistics (Spearman test and Principal Component Analysis) were implemented. Basic results: Female gender and traveling abroad for sport, the desire of distinction in science and the social selectiveness tend to protect against bullying. High semester, tallness, high educational influence of family and cyber-bullying increase the susceptibility to victimization. These who have experienced bullying as children still tend to experience exclusion. Libeling may even be a reason for not attending lectures. High grade seems not only to protect but also to discourage someone from practicing bullying. Science ambitions seem to be related with elitist ideology, unlike professional ambitions which seem to be related with humanism or sociability. Social selectiveness based on politeness and friendliness also retains the practicing of bullying. Five types of bullying targets have been depicted: "full victim", "apprenticed", "libeled scapegoat", "ridiculed scapegoat" and "gladiator". Three types of practicing bullying are formulated: "stimulating victimizer", "provocateur" and "egoist inspirator".
\end{abstract}

\section{Keywords}

Bullying, Social Network Analysis, Typology

\section{Introduction}

Bullying can be regarded as a form of destructive relation, including also verbal aggressiveness. While verbal aggressiveness is a form of destructive communication, bullying is extended beyond verbal attacks, even to physical violence. Ver- 
bal aggressiveness has been extensively explored [1]-[15]. The exploration of bullying is an indispensable step to integrate the research approach to the general phenomenon of aggressiveness in the education system. Bullying is defined as a form of attack, in which a behavior has as purpose to disrupt or disturb, is presented repeatedly and there is a force imbalance as a more powerful person attacks a less powerful one [16]. Bullying can be carried out by physical contact, words, or other ways, such as obscene gestures, and intentional exclusion from a group. In a British study, it was found that direct verbal aggression was the most common form of bullying [17]. According to [18], bully victims had higher levels of psychological distress, low self-esteem, more unhealthy behaviors and less support from parents and teachers. Additionally, the male students seem to be more often involved in aggressive communication as assailant or victim [19]. [20] supported that most high levels of bullying which are mostly associated with male students at schools, lower levels of empathy, and belong to the most authoritarian or violent families. Bullies presented a higher level of externalization, while victims more internalizing symptoms [21]. In fact, bullies usually motivate others against the target, and the identities of the perpetrators are often known to them [22]. Boys are more intimidated than girls while the family income appears to be negatively related to bullying [23]. It has been supported that the perception of bullying is significantly association with levels of shyness, the confidence and satisfaction with friendships [24]. [25] investigated bullying among undergraduate students showing that $24.7 \%$ had seen incidents of bullying among students occasionally while $2.8 \%$ very often. The effects of bullying both for victims and for bullies are continuing long after the years of university [26]. [27] concluded that the victimization leads to higher levels of depressive tendencies. According to [28], experiences of victimization at high school affect the transition to university. [29] found that former bullies are more likely to show criminal behavior at the age of 24 . The effects of victimization on the personality of a child are more severe, especially when the victimization occurs at very early age [30]. This study concluded that there was an obvious correlation between experiences of victimization at school, and difficulty of adaptation to university [31].

As for the deeper theoretical background (system theory operationalized in terms of social network analysis), several studies have been conducted until now [32]-[37]. These studies have focused on verbal aggressiveness and not on bullying. Nevertheless, bullying is also a relation measurable like verbal aggressiveness. Thus, bullying can also be explored as a social structure/hierarchy (who concentrates most bullying actions exerted by others, what chains of successive bullying actions are shaped and who is the final receiver of these). Therefore, network analysis (constituting both theory as an empirically applicable form of system theory and a method used for detecting and measuring relations as social structure) has been selected as an appropriate approach in this research.

\section{Aim and Innovation}

The present study is aiming at: 1) detecting determinants of shaping bullying 
structures among students in classes of university departments and 2) formulating typology (behavioral patterns) of bullying target and bullies. Previous analyses (e.g. [38]-[42]) have suggested similar approaches to the determinants and behavioral patterns focusing, however, on verbal aggressiveness. This research endeavors to disclose such structural types and determinants of bullying, beyond common verbal aggressiveness. The application of a tested method (network analysis) in bullying constitutes the innovation of this study.

\section{Method}

Four classes of the University of Thessaly has been selected for network sampling: two classes of the department of Veterinary $\left(\mathrm{N} 1=66\right.$ of the $4^{\text {th }}$ semestermale $=23$, female $=43, \mathrm{~N} 2=53$ of the $8^{\text {th }}$ semester-male $=21$, female $\left.=32\right)$ and two classes of the department of Physical Education and Sport Sciences $(\mathrm{N} 3=57$ of the $4^{\text {th }}$ semester-male $=29$, female $=28, N 4=42$ of the $8^{\text {th }}$ semester-male $=19$, female $=23$ ) (namely, 218 nodes in total). These particular classes were selected in order to consider the variable of study time (semester) as well as the empirical data appearing in Veterinary which is a department quite different from the Physical Education in terms of corporal competitiveness. If the research focused only on Physical Education department, this could be criticized as biased because Physical Education could be regarded as a field quite susceptible to bullying due to its content and context (competitiveness and corporal force in sport games). Simultaneously, this additional department was Veterinary and not e.g. Humanities (that strongly focus on cultural discourse and intellectual activity which could be supposed to "refine" the everyday communication and mitigate bullying), as Veterinary also belongs to the wide area of positivist fields and thus it is not fully unfamiliar to Physical Education. Thus, the whole sample is not so biased as it would be if it consisted only of Physical Education students, and also not so heterogeneous if it contained Humanities. Subsequently, it is a judgment sample and not a random one, but this is not a weakness as it aims at analytic and not descriptive statistics. Research ethics were observed and discretion was guaranteed so that participants gave sincere answers.

The network part of the questionnaire was similar to previous tested questionnaires [33] [36] [43]. Questions relevant to bullying were reformulated from psychometric into network form. The non-network part contained individual parameters related to the personal profile of students (e.g. socio-economic state, age, gender).

Network variables (indegree, Katz status, pagerank, authority and outdegree) were calculated by Visone 1.1 in order to enable a multiple structural approach. Cross-sectional analysis (spearman test) has been applied in order to detect correlations between non-network and network variables. This test was preferred to multivariate analysis, as it enables an overview on all possible relations [33] [41] [42]. Principal Component Analysis was used for revealing behavioral patterns (typology). 


\section{Results and Discussion}

In Diagram 1, examples of bullying social networks from two departments of the University of Thessaly are presented. The densest one (1.3\%) is this of causing unhappiness while the other two ones (exclusion from the companionship and fight) are much thinner (0.05\%). This is understandable as the former one consists of a bullying form quite simple and usual while fight and exclusion at students' age is rarer.

In Table 1, female students seem to be protected against being a target of causing disagreements. The same applies also in students of high semesters in general but these seem to be involved in scolding encouraged by others. Students of specific physical qualification (tallness) tend to become a target of causing disagreements or encouraged scolding. Students with mother of high education level appear also to become target of causing disagreements. These who achieve to obtain high grade appear to become target of causing disagreements but to avoid being involved in scolding encouraged by others. Tendency to be involved in such scolding characterizes also these who travel abroad for sport reasons.

These who consider themselves to positively inspire their students regarding

Diagram 1. Examples of bullying social networks among students at departments (Veterinary and Physical Education) at the University of Thessaly, 2017.

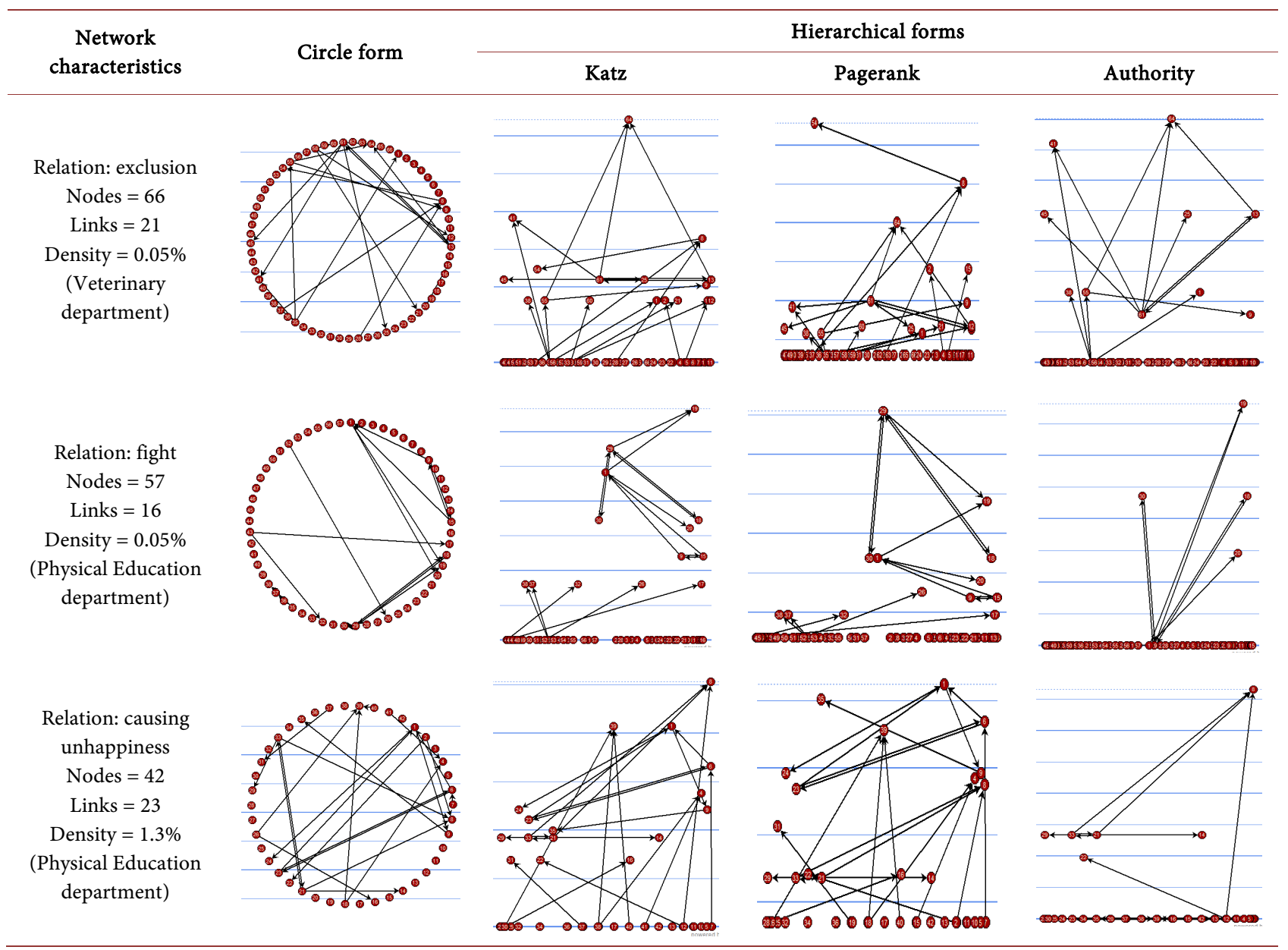


Table 1. Detecting parameters of bullying targeting through different network indicators (disagreement and scold).

\begin{tabular}{|c|c|c|c|c|c|c|c|c|c|c|c|}
\hline & $\begin{array}{l}\text { Male }=0 \\
\text { female }=1\end{array}$ & Semester & Height & $\begin{array}{c}\text { Education } \\
\text { level of } \\
\text { mother }\end{array}$ & $\begin{array}{l}\text { General } \\
\text { grade }\end{array}$ & $\begin{array}{c}\text { Travel } \\
\text { abroad } \\
\text { for sport } \\
\text { reasons }\end{array}$ & $\begin{array}{l}\text { Inspiring } \\
\text { positively } \\
\text { in courses }\end{array}$ & $\begin{array}{l}\text { Desire for } \\
\text { distinction } \\
\text { in science }\end{array}$ & $\begin{array}{l}\text { Practiced } \\
\text { cyber-bullying } \\
\text { to students }\end{array}$ & $\begin{array}{l}\text { Practiced } \\
\text { bullying } \\
\text { as a } \\
\text { child }\end{array}$ & $\begin{array}{l}\text { Cleverness } \\
\text { as } \\
\text { criterion } \\
\text { for } \\
\text { friendship }\end{array}$ \\
\hline \multirow[t]{2}{*}{$\begin{array}{c}\text { Causing } \\
\text { disagreements } \\
\text { (indegree) }\end{array}$} & -0.073 & -0.125 & 0.096 & $\begin{array}{c}0.218 \\
(*)\end{array}$ & -0.091 & 0.019 & -0.060 & 0.031 & 0.114 & 0.172 & $\begin{array}{c}-0.296 \\
(* *)\end{array}$ \\
\hline & 0.281 & 0.210 & 0.337 & 0.027 & 0.375 & 0.850 & 0.548 & 0.759 & 0.252 & 0.083 & 0.002 \\
\hline \multirow[t]{2}{*}{$\begin{array}{c}\text { Causing } \\
\text { disagreements } \\
\text { (status) }\end{array}$} & -0.073 & -0.126 & 0.097 & $\begin{array}{c}0.222 \\
(*)\end{array}$ & -0.093 & 0.013 & -0.059 & 0.032 & 0.114 & 0.170 & $\begin{array}{c}-0.297 \\
(* *)\end{array}$ \\
\hline & 0.283 & 0.208 & 0.331 & 0.025 & 0.362 & 0.894 & 0.555 & 0.749 & 0.252 & 0.085 & 0.002 \\
\hline \multirow[t]{2}{*}{$\begin{array}{c}\text { Causing } \\
\text { disagreements } \\
\text { (pagerank) }\end{array}$} & $-0.152\left(^{*}\right)$ & $\begin{array}{c}-0.344 \\
(* *)\end{array}$ & $\begin{array}{c}0.228 \\
(*)\end{array}$ & -0.026 & $\begin{array}{c}0.212 \\
\left({ }^{*}\right)\end{array}$ & 0.159 & -0.087 & $\begin{array}{c}-0.226 \\
(*)\end{array}$ & 0.083 & 0.138 & $\begin{array}{c}-0.344 \\
(* *)\end{array}$ \\
\hline & 0.025 & 0.000 & 0.021 & 0.795 & 0.037 & 0.110 & 0.384 & 0.022 & 0.404 & 0.165 & 0.000 \\
\hline \multirow[t]{2}{*}{$\begin{array}{c}\text { Causing } \\
\text { disagreements } \\
\text { (authority) }\end{array}$} & -0.009 & -0.009 & 0.072 & 0.050 & -0.128 & 0.072 & $\begin{array}{c}-0.196 \\
\left({ }^{*}\right)\end{array}$ & -0.010 & $\begin{array}{c}0.222 \\
\left({ }^{*}\right)\end{array}$ & $\begin{array}{c}0.247 \\
\left({ }^{*}\right)\end{array}$ & $\begin{array}{c}-0.226 \\
(*)\end{array}$ \\
\hline & 0.898 & 0.929 & 0.472 & 0.619 & 0.213 & 0.470 & 0.047 & 0.922 & 0.024 & 0.012 & 0.021 \\
\hline \multirow[t]{2}{*}{$\begin{array}{l}\text { Encouraging to } \\
\text { scold (indegree) }\end{array}$} & 0.000 & $\begin{array}{c}0.555 \\
(* *)\end{array}$ & 0.051 & 0.124 & $\begin{array}{c}-0.298 \\
(* *)\end{array}$ & $\begin{array}{c}-0.196 \\
(*)\end{array}$ & 0.061 & 0.129 & 0.088 & -0.100 & 0.011 \\
\hline & 0.999 & 0.000 & 0.609 & 0.212 & 0.003 & 0.047 & 0.541 & 0.193 & 0.377 & 0.314 & 0.912 \\
\hline \multirow[t]{2}{*}{$\begin{array}{l}\text { Encouraging } \\
\text { to scold (katz) }\end{array}$} & 0.000 & $\begin{array}{c}0.555 \\
(* *)\end{array}$ & 0.051 & 0.124 & $\begin{array}{c}-0.298 \\
(* *)\end{array}$ & $\begin{array}{c}-0.196 \\
(*)\end{array}$ & 0.061 & 0.129 & 0.088 & -0.100 & 0.011 \\
\hline & 0.999 & 0.000 & 0.609 & 0.212 & 0.003 & 0.047 & 0.541 & 0.193 & 0.377 & 0.314 & 0.912 \\
\hline \multirow[t]{2}{*}{$\begin{array}{l}\text { Encouraging to } \\
\text { scold (pagerank) }\end{array}$} & -0.010 & $\begin{array}{c}0.495 \\
(* *)\end{array}$ & 0.077 & -0.043 & -0.013 & -0.032 & 0.107 & 0.128 & -0.003 & -0.080 & -0.096 \\
\hline & 0.880 & 0.000 & 0.440 & 0.668 & 0.901 & 0.748 & 0.281 & 0.198 & 0.974 & 0.421 & 0.334 \\
\hline \multirow[t]{2}{*}{$\begin{array}{l}\text { Encouraging to } \\
\text { scold (authority) }\end{array}$} & 0.000 & $\begin{array}{c}0.555 \\
(* *)\end{array}$ & 0.051 & 0.124 & $\begin{array}{c}-0.298 \\
(* *)\end{array}$ & $\begin{array}{c}-0.196 \\
(*)\end{array}$ & 0.061 & 0.129 & 0.088 & -0.100 & 0.011 \\
\hline & 0.999 & 0.000 & 0.609 & 0.212 & 0.003 & 0.047 & 0.541 & 0.193 & 0.377 & 0.314 & 0.912 \\
\hline
\end{tabular}

studies tend also to be protected against becoming a target of causing disagreements. The same also applies in case if these who are interested in scientific distinction. Students who committed bullying through internet become also target of causing disagreements. These who are selective in friendly companionship tend to avoid being targeted of causing disagreements.

The afore-mentioned results revealed that certain properties such as the female gender, the high grade and the traveling abroad for sport, the capacity of inspiring interest in studies and the desire of distinction in science and the selectiveness in friend companion mainly tend to protect against causing of disagreements. This is understandable as these properties are connected with restricted susceptibility to conflicts, with persuasiveness and reputation as well as 
avoidance of incompatible friends.

Other properties such as high semester, tallness, high educational influence of family and having practiced cyber-bullying are mainly connected with becoming target of being involved in disagreements or scold triggered by others. This can be attributed either to jealousness or to their general tendency to implicate in bullying. The high semester increases the involvement in scold, as the long-familiarized students find easier to participate in conflicts. Similar studies have also taken place in verbal aggressiveness [41] [42].

The results of Table 2 are quite similar to those of Table 1. Tallness family education background (father instead of mother this time), the course of study time and the subsequent familiarization with students milieus seem to increase the involvement in bullying. On the other hand, particular criteria of selecting friends protect against bullying. Additionally, weight seems also to increase targeting of bullying, as it is related with fatness.

In Table 3, characteristics like weight and family education background have

Table 2. Detecting parameters of bullying targeting through different network indicators (roasting and unhappiness).

\begin{tabular}{|c|c|c|c|c|c|c|}
\hline & Height & Weight & $\begin{array}{c}\text { Education } \\
\text { level of } \\
\text { father }\end{array}$ & Semester & $\begin{array}{l}\text { Cleverness as } \\
\text { criterion for } \\
\text { friendship }\end{array}$ & $\begin{array}{l}\text { Politeness as } \\
\text { criterion for } \\
\text { friendship }\end{array}$ \\
\hline \multirow[t]{2}{*}{$\begin{array}{c}\text { Roasting } \\
\text { (indegree) }\end{array}$} & $\begin{array}{c}0.274 \\
(* *)\end{array}$ & $\begin{array}{c}0.284 \\
(* *)\end{array}$ & $\begin{array}{c}0.215 \\
(*)\end{array}$ & -0.013 & 0.020 & $\begin{array}{c}-0.278 \\
(* *)\end{array}$ \\
\hline & 0.005 & 0.004 & 0.030 & 0.894 & 0.839 & 0.005 \\
\hline \multirow[t]{2}{*}{$\begin{array}{l}\text { Roasting } \\
\text { (status) }\end{array}$} & $\begin{array}{c}0.278 \\
(* *)\end{array}$ & $\begin{array}{c}0.277 \\
(* *)\end{array}$ & $\begin{array}{c}0.201 \\
(*)\end{array}$ & -0.022 & 0.007 & $\begin{array}{c}-0.280 \\
(* *)\end{array}$ \\
\hline & 0.005 & 0.005 & 0.043 & 0.827 & 0.941 & 0.004 \\
\hline \multirow[t]{2}{*}{$\begin{array}{c}\text { Roasting } \\
\text { (pagerank) }\end{array}$} & 0.137 & 0.190 & 0.187 & $\begin{array}{c}0.273 \\
(* *)\end{array}$ & -0.084 & $\begin{array}{c}-0.199 \\
(*)\end{array}$ \\
\hline & 0.169 & 0.057 & 0.060 & 0.006 & 0.400 & 0.043 \\
\hline \multirow[t]{2}{*}{$\begin{array}{c}\text { Roasting } \\
\text { (authority) }\end{array}$} & $\begin{array}{c}0.320 \\
(* *)\end{array}$ & $\begin{array}{c}0.240 \\
(*)\end{array}$ & 0.101 & 0.039 & 0.009 & $\begin{array}{c}-0.214 \\
(*)\end{array}$ \\
\hline & 0.001 & 0.016 & 0.312 & 0.696 & 0.927 & 0.030 \\
\hline \multirow[t]{2}{*}{$\begin{array}{c}\text { Causing } \\
\text { unhappiness } \\
\text { (indegree) }\end{array}$} & -0.080 & -0.156 & -0.070 & 0.035 & $\begin{array}{c}-0.253 \\
(* *)\end{array}$ & -0.185 \\
\hline & 0.423 & 0.120 & 0.482 & 0.725 & 0.010 & 0.061 \\
\hline \multirow[t]{2}{*}{$\begin{array}{c}\text { Causing } \\
\text { unhappiness } \\
\text { (status) }\end{array}$} & -0.080 & -0.159 & -0.072 & 0.030 & $\begin{array}{c}-0.256 \\
(* *)\end{array}$ & -0.185 \\
\hline & 0.424 & 0.113 & 0.470 & 0.767 & 0.009 & 0.061 \\
\hline \multirow[t]{2}{*}{$\begin{array}{c}\text { Causing } \\
\text { unhappiness } \\
\text { (pagerank) }\end{array}$} & 0.011 & -0.043 & -0.141 & 0.186 & $\begin{array}{c}-0.203 \\
(*)\end{array}$ & -0.098 \\
\hline & 0.910 & 0.671 & 0.157 & 0.061 & 0.040 & 0.323 \\
\hline
\end{tabular}


Table 3. Detecting parameters of bullying targeting through different network indicators (harassment and exclusion).

\begin{tabular}{|c|c|c|c|c|c|c|c|c|c|c|c|c|}
\hline & $\begin{array}{l}\frac{5}{50} \\
.000 \\
3\end{array}$ & 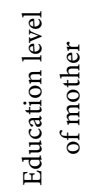 & 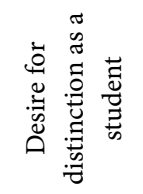 & 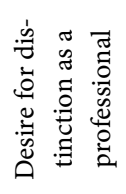 & 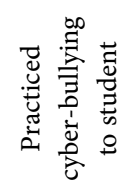 & 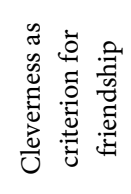 & 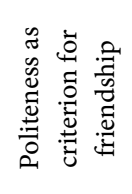 & 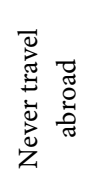 & 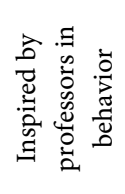 & 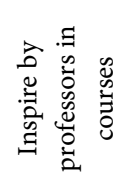 & 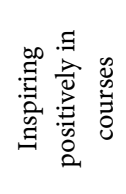 & 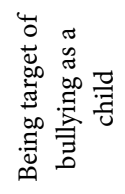 \\
\hline \multirow[t]{2}{*}{$\begin{array}{c}\text { Harassment } \\
\text { (indegree) }\end{array}$} & 0.023 & 0.014 & 0.012 & -0.050 & 0.111 & $\begin{array}{c}-0.207 \\
(*)\end{array}$ & -0.106 & $\begin{array}{c}0.199 \\
\left({ }^{*}\right)\end{array}$ & -0.012 & -0.019 & 0.037 & -0.004 \\
\hline & 0.819 & 0.885 & 0.902 & 0.617 & 0.263 & 0.036 & 0.289 & 0.044 & 0.905 & 0.850 & 0.709 & 0.968 \\
\hline \multirow[t]{2}{*}{$\begin{array}{l}\text { Harassment } \\
\text { (status) }\end{array}$} & 0.060 & -0.031 & 0.008 & 0.021 & 0.028 & $\begin{array}{c}-0.199 \\
(*)\end{array}$ & 0.038 & $\begin{array}{c}0.228 \\
\left({ }^{*}\right)\end{array}$ & -0.030 & -0.116 & -0.009 & -0.102 \\
\hline & 0.550 & 0.755 & 0.934 & 0.834 & 0.780 & 0.044 & 0.702 & 0.020 & 0.763 & 0.242 & 0.924 & 0.307 \\
\hline \multirow[t]{2}{*}{$\begin{array}{c}\text { Harassment } \\
\text { (pagerank) }\end{array}$} & 0.071 & -0.186 & 0.108 & 0.045 & -0.037 & $\begin{array}{c}-0.243 \\
\left({ }^{*}\right)\end{array}$ & 0.017 & $\begin{array}{c}0.294 \\
(* *)\end{array}$ & -0.103 & $\begin{array}{c}-0.256 \\
(* *)\end{array}$ & -0.083 & -0.120 \\
\hline & 0.479 & 0.060 & 0.279 & 0.651 & 0.709 & 0.013 & 0.862 & 0.003 & 0.301 & 0.009 & 0.407 & 0.228 \\
\hline \multirow[t]{2}{*}{$\begin{array}{c}\text { Harassment } \\
\text { (authority) }\end{array}$} & 0.093 & 0.095 & 0.022 & 0.077 & 0.115 & -0.113 & -0.052 & $\begin{array}{c}0.240 \\
\left({ }^{*}\right)\end{array}$ & -0.012 & -0.053 & 0.054 & -0.192 \\
\hline & 0.354 & 0.342 & 0.822 & 0.440 & 0.248 & 0.257 & 0.601 & 0.015 & 0.904 & 0.598 & 0.589 & 0.053 \\
\hline \multirow[t]{2}{*}{$\begin{array}{l}\text { Exclusion } \\
\text { (indegree) }\end{array}$} & $\begin{array}{c}0.242 \\
(*)\end{array}$ & $\begin{array}{c}0.212 \\
(*)\end{array}$ & $-0.194\left(^{*}\right)$ & $\begin{array}{c}-0.287 \\
(* *)\end{array}$ & $\begin{array}{c}0.393 \\
(* *)\end{array}$ & -0.098 & $\begin{array}{c}-0.334 \\
(* *)\end{array}$ & -0.028 & 0.134 & $\begin{array}{c}0.247 \\
(*)\end{array}$ & 0.172 & 0.162 \\
\hline & 0.015 & 0.032 & 0.049 & 0.003 & 0.000 & 0.324 & 0.001 & 0.778 & 0.179 & 0.012 & 0.082 & 0.101 \\
\hline \multirow[t]{2}{*}{$\begin{array}{c}\text { Exclusion } \\
\text { (status) }\end{array}$} & $\begin{array}{c}0.229 \\
(*)\end{array}$ & $\begin{array}{c}0.215 \\
(*)\end{array}$ & -0.179 & $\begin{array}{c}-0.282 \\
(* *)\end{array}$ & $\begin{array}{c}0.383 \\
(* *)\end{array}$ & -0.108 & $\begin{array}{c}-0.325 \\
(* *)\end{array}$ & -0.020 & 0.128 & $\begin{array}{c}0.244 \\
\left({ }^{*}\right)\end{array}$ & 0.171 & 0.165 \\
\hline & 0.021 & 0.029 & 0.070 & 0.004 & 0.000 & 0.280 & 0.001 & 0.840 & 0.199 & 0.013 & 0.084 & 0.095 \\
\hline \multirow[t]{2}{*}{$\begin{array}{l}\text { Exclusion } \\
\text { (pagerank) }\end{array}$} & 0.113 & 0.042 & -0.110 & -0.074 & 0.189 & -0.138 & -0.158 & -0.013 & 0.177 & $\begin{array}{c}0.236 \\
\left({ }^{*}\right)\end{array}$ & 0.170 & 0.132 \\
\hline & 0.261 & 0.676 & 0.267 & 0.458 & 0.056 & 0.166 & 0.112 & 0.898 & 0.074 & 0.017 & 0.087 & 0.183 \\
\hline \multirow[t]{2}{*}{$\begin{array}{l}\text { Exclusion } \\
\text { (authority) }\end{array}$} & 0.161 & 0.061 & -0.124 & $\begin{array}{c}-0.331 \\
(* *)\end{array}$ & 0.099 & -0.127 & $\begin{array}{c}-0.294 \\
(* *)\end{array}$ & -0.068 & $\begin{array}{c}0.279 \\
(* *)\end{array}$ & $\begin{array}{c}0.304 \\
(* *)\end{array}$ & $\begin{array}{c}0.220 \\
\left({ }^{*}\right)\end{array}$ & $\begin{array}{c}0.199 \\
(*)\end{array}$ \\
\hline & 0.109 & 0.542 & 0.211 & 0.001 & 0.320 & 0.200 & 0.003 & 0.494 & 0.004 & 0.002 & 0.026 & 0.044 \\
\hline
\end{tabular}

also been explored in previous studies [33] [40] [44]. They seem to lead to victimization. The desire for distinction (in this case, not in science, but in studying or in professional career), the involvement in cyber-bullying and the friends selectiveness appear once again to protect against or to trigger bullying in a sense similar to what is discussed in the tables above. Additionally, the absolute lack of traveling abroad seems to attract bullying, as it signifies poorness at social and mentality level. These who let themselves be inspired by professors mainly become target of bullying, as they tend be characterized by quite different mentality. Moreover, inspiring (or believing that someone inspires) others may leads up to annoyance and subsequently to exclusion. Finally, these who have experienced bullying as children, they still tend to experience exclusion. This reveals that there may be permanent attributes attracting bullying on certain persons.

In Table 4, the role of family education influence, having committed cyber-bullying to others, the desire for distinction, the friends selectiveness seems 
Table 4. Detecting parameters of bullying targeting through different network indicators (libeling and fight).

\begin{tabular}{|c|c|c|c|c|c|c|c|}
\hline & 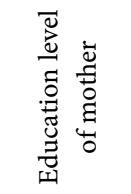 & 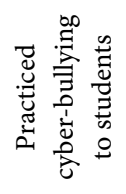 & 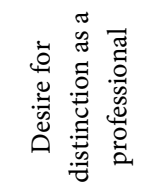 & 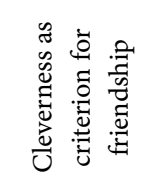 & 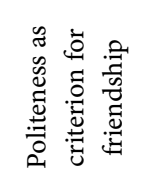 & 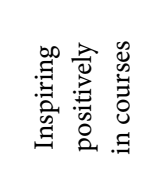 & 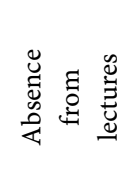 \\
\hline \multirow[t]{2}{*}{$\begin{array}{l}\text { Libeling } \\
\text { (indegree) }\end{array}$} & -0.008 & $0.219\left(^{*}\right)$ & -0.166 & $-0.309(* *)$ & $-0.213\left(^{*}\right)$ & -0.131 & $-0.195\left(^{*}\right)$ \\
\hline & 0.935 & 0.026 & 0.094 & 0.002 & 0.031 & 0.187 & 0.048 \\
\hline \multirow[t]{2}{*}{$\begin{array}{l}\text { Libeling } \\
\text { (status) }\end{array}$} & -0.008 & $0.218\left(^{*}\right)$ & -0.166 & $-0.309(* *)$ & $-0.213\left(^{*}\right)$ & -0.130 & $-0.195\left(^{*}\right)$ \\
\hline & 0.935 & 0.027 & 0.094 & 0.002 & 0.031 & 0.189 & 0.049 \\
\hline \multirow[t]{2}{*}{$\begin{array}{c}\text { Libeling } \\
\text { (pagerank) }\end{array}$} & -0.167 & 0.080 & -0.074 & $-0.231\left(^{*}\right)$ & -0.138 & -0.109 & -0.113 \\
\hline & 0.092 & 0.423 & 0.459 & 0.019 & 0.166 & 0.275 & 0.254 \\
\hline \multirow[t]{2}{*}{$\begin{array}{l}\text { Libeling } \\
\text { (authority) }\end{array}$} & -0.100 & $0.236\left(^{*}\right)$ & -0.167 & $-0.226\left(^{*}\right)$ & -0.018 & $-0.262(* *)$ & $-0.209\left(^{*}\right)$ \\
\hline & 0.314 & 0.016 & 0.092 & 0.022 & 0.855 & 0.008 & 0.034 \\
\hline \multirow[t]{2}{*}{$\begin{array}{c}\text { Fight } \\
\text { (indegree) }\end{array}$} & $0.245\left(^{*}\right)$ & 0.127 & $-0.241\left(^{*}\right)$ & $-0.282(* *)$ & -0.023 & 0.001 & 0.096 \\
\hline & 0.013 & 0.202 & 0.014 & 0.004 & 0.820 & 0.991 & 0.336 \\
\hline \multirow[t]{2}{*}{$\begin{array}{l}\text { Fight } \\
\text { (status) }\end{array}$} & $0.250\left(^{*}\right)$ & 0.123 & $-0.250\left(^{*}\right)$ & $-0.284(* *)$ & -0.028 & 0.007 & 0.093 \\
\hline & 0.011 & 0.217 & 0.011 & 0.004 & 0.781 & 0.942 & 0.353 \\
\hline \multirow[t]{2}{*}{$\begin{array}{c}\text { Fight } \\
\text { (pagerank) }\end{array}$} & 0.064 & 0.010 & -0.140 & $-0.261(* *)$ & -0.027 & 0.047 & -0.041 \\
\hline & 0.522 & 0.917 & 0.159 & 0.008 & 0.787 & 0.637 & 0.684 \\
\hline $\begin{array}{c}\text { Fight } \\
\text { (authority) }\end{array}$ & $0.267^{(* *)}$ & 0.045 & $-0.322\left({ }^{* *}\right)$ & $-0.211\left(^{*}\right)$ & -0.025 & 0.051 & 0.088 \\
\hline & 0.006 & 0.651 & 0.001 & 0.033 & 0.799 & 0.608 & 0.374 \\
\hline
\end{tabular}

to be similar to the afore-mentioned results. The positive inspiration of others (in this case, in behavior) seems also to be in this case positively connected with protection against bullying. Inversely, due to lack of bullying one perceives his influence as positive inspiration. Additionally, being a target of libeling may even a reason for not attending lectures.

In Table 5, not targeting but practicing bullying is examined. Gender has also been explored in previous studies [45] [46]. The female students appear to avoid practicing bullying. Tallness and weight prove to be not only a determinant of targeting, as discussed above, but also a parameter of practicing bullying, as a return. The same applies also for the education background of family and the study time. Students with high grade tend not only to be protected against but also not to practice bullying. These who desire distinction in science tend to 
Table 5. Detecting parameters of practicing bullying (outdegree) through different network indicators (libeling and fight).

\begin{tabular}{|c|c|c|c|c|c|c|c|c|c|c|c|c|c|c|c|}
\hline & $\begin{array}{l}\dot{\bar{v}} \\
\vec{\Xi} \\
\stackrel{\Xi}{0}\end{array}$ & 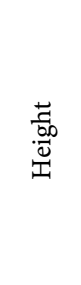 & $\begin{array}{l}\frac{7}{50} \\
\frac{500}{00} \\
3\end{array}$ & 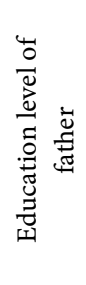 & 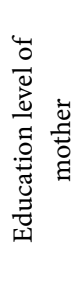 & 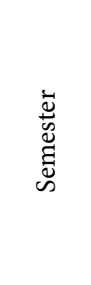 & 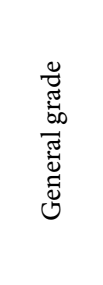 & 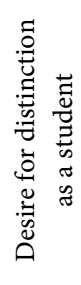 & 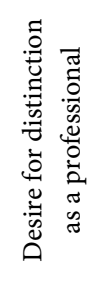 & 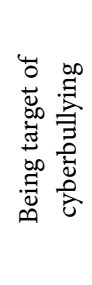 & 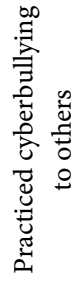 & 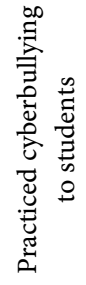 & 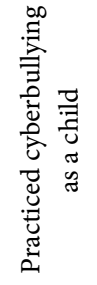 & 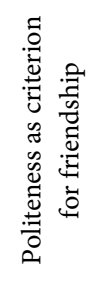 & 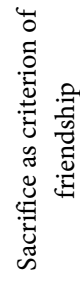 \\
\hline \multirow[t]{2}{*}{$\begin{array}{c}\text { Making fun on } \\
\text { others' cost } \\
\text { (outdegree) }\end{array}$} & -0.094 & 0.138 & 0.102 & 0.121 & 0.116 & -0.092 & 0.144 & 0.184 & -0.021 & 0.118 & $\begin{array}{c}0.220 \\
(*)\end{array}$ & $\begin{array}{c}0.207 \\
(*)\end{array}$ & 0.101 & -0.095 & $\begin{array}{c}0.260 \\
(* *)\end{array}$ \\
\hline & 0.168 & 0.166 & 0.310 & 0.225 & 0.244 & 0.360 & 0.158 & 0.063 & 0.829 & 0.233 & 0.025 & 0.036 & 0.309 & 0.339 & 0.008 \\
\hline \multirow[t]{2}{*}{$\begin{array}{c}\text { Causing } \\
\text { unhappiness } \\
\text { (outdegree) }\end{array}$} & $\begin{array}{c}-0.215 \\
(* *)\end{array}$ & $\begin{array}{c}0.224 \\
(*)\end{array}$ & $\begin{array}{c}0.203 \\
\left(^{*}\right)\end{array}$ & 0.129 & $\begin{array}{c}0.206 \\
\left(^{*}\right)\end{array}$ & 0.086 & -0.043 & 0.069 & 0.006 & -0.121 & $\begin{array}{c}0.258 \\
(* *)\end{array}$ & 0.144 & 0.083 & -0.093 & $\begin{array}{c}0.308 \\
(* *)\end{array}$ \\
\hline & 0.001 & 0.024 & 0.042 & 0.195 & 0.037 & 0.391 & 0.674 & 0.488 & 0.956 & 0.224 & 0.009 & 0.147 & 0.407 & 0.350 & 0.002 \\
\hline \multirow[t]{2}{*}{$\begin{array}{l}\text { Harassment } \\
\text { (outdegree) }\end{array}$} & $\begin{array}{c}-0.221 \\
(* *)\end{array}$ & 0.159 & $\begin{array}{c}0.221 \\
\left(^{*}\right)\end{array}$ & 0.129 & 0.076 & 0.080 & -0.119 & $\begin{array}{c}0.241 \\
(*)\end{array}$ & -0.047 & 0.021 & $\begin{array}{c}0.287 \\
(* *)\end{array}$ & 0.188 & 0.149 & -0.089 & 0.118 \\
\hline & 0.001 & 0.110 & 0.027 & 0.198 & 0.445 & 0.426 & 0.247 & 0.014 & 0.637 & 0.831 & 0.003 & 0.057 & 0.133 & 0.369 & 0.239 \\
\hline \multirow[t]{2}{*}{$\begin{array}{l}\text { Exclusion } \\
\text { (outdegree) }\end{array}$} & -0.103 & 0.126 & 0.066 & 0.087 & 0.147 & 0.039 & 0.061 & $\begin{array}{c}0.282 \\
(* *)\end{array}$ & -0.120 & 0.125 & $\begin{array}{c}0.194 \\
(*)\end{array}$ & 0.192 & 0.131 & -0.086 & 0.054 \\
\hline & 0.128 & 0.206 & 0.514 & 0.387 & 0.137 & 0.697 & 0.555 & 0.004 & 0.229 & 0.207 & 0.050 & 0.052 & 0.187 & 0.389 & 0.589 \\
\hline \multirow[t]{2}{*}{$\begin{array}{c}\text { Spread rumors } \\
\text { (outdegree) }\end{array}$} & $\begin{array}{c}-0.183 \\
(* *)\end{array}$ & $\begin{array}{c}0.210 \\
\left({ }^{*}\right)\end{array}$ & $\begin{array}{c}0.247 \\
\left(^{*}\right)\end{array}$ & $\begin{array}{c}0.262 \\
(* *)\end{array}$ & 0.159 & 0.005 & -0.021 & 0.114 & 0.007 & 0.113 & $\begin{array}{c}0.278 \\
(* *)\end{array}$ & $\begin{array}{c}0.270 \\
(* *)\end{array}$ & 0.105 & -0.159 & 0.129 \\
\hline & 0.007 & 0.034 & 0.013 & 0.008 & 0.108 & 0.958 & 0.837 & 0.251 & 0.943 & 0.254 & 0.004 & 0.006 & 0.291 & 0.108 & 0.196 \\
\hline \multirow[t]{2}{*}{$\begin{array}{c}\text { Fight } \\
\text { (outdegree) }\end{array}$} & $\begin{array}{c}-0.168 \\
\left({ }^{*}\right)\end{array}$ & 0.194 & 0.154 & 0.094 & 0.093 & -0.139 & 0.019 & 0.026 & $\begin{array}{c}-0.211 \\
\left.{ }^{*}\right)\end{array}$ & $\begin{array}{c}0.253 \\
\left(^{*}\right)\end{array}$ & $\begin{array}{c}0.282 \\
(* *)\end{array}$ & $\begin{array}{c}0.211 \\
\left({ }^{*}\right)\end{array}$ & 0.101 & $\begin{array}{c}-0.265 \\
(* *)\end{array}$ & $\begin{array}{c}0.293 \\
(* *)\end{array}$ \\
\hline & 0.013 & 0.051 & 0.124 & 0.347 & 0.351 & 0.163 & 0.851 & 0.791 & 0.032 & 0.010 & 0.004 & 0.033 & 0.309 & 0.007 & 0.003 \\
\hline \multirow[t]{2}{*}{$\begin{array}{c}\text { Causing } \\
\text { disagreements } \\
\text { (outdegree) }\end{array}$} & $\begin{array}{c}-0.199 \\
(* *)\end{array}$ & $\begin{array}{c}0.288 \\
(* *)\end{array}$ & $\begin{array}{c}0.233 \\
(*)\end{array}$ & 0.193 & $\begin{array}{c}0.229 \\
\left({ }^{*}\right)\end{array}$ & 0.033 & -0.002 & 0.118 & -0.115 & 0.030 & $\begin{array}{c}0.346 \\
(* *)\end{array}$ & $\begin{array}{c}0.266 \\
(* *)\end{array}$ & $\begin{array}{c}0.281 \\
(* *)\end{array}$ & -0.109 & $\begin{array}{c}0.259 \\
(* *)\end{array}$ \\
\hline & 0.003 & 0.003 & 0.019 & 0.051 & 0.020 & 0.739 & 0.984 & 0.235 & 0.246 & 0.765 & 0.000 & 0.007 & 0.004 & 0.275 & 0.009 \\
\hline \multirow[t]{2}{*}{$\begin{array}{c}\text { Encouraging } \\
\text { to scold } \\
\text { (outdegree) }\end{array}$} & -0.077 & 0.152 & $\begin{array}{c}0.269 \\
(* *)\end{array}$ & $\begin{array}{c}0.212 \\
(*)\end{array}$ & 0.123 & $\begin{array}{c}0.536 \\
(* *)\end{array}$ & $\begin{array}{c}-0.201 \\
\left({ }^{*}\right)\end{array}$ & $\begin{array}{c}0.237 \\
\left(^{*}\right)\end{array}$ & -0.009 & $\begin{array}{c}0.238 \\
(*)\end{array}$ & 0.192 & $\begin{array}{c}0.264 \\
(* *)\end{array}$ & -0.007 & -0.131 & 0.135 \\
\hline & 0.257 & 0.126 & 0.007 & 0.033 & 0.215 & 0.000 & 0.048 & 0.016 & 0.929 & 0.016 & 0.051 & 0.007 & 0.941 & 0.186 & 0.178 \\
\hline
\end{tabular}

practice bullying, although they are protected against it. This asymmetric relation can be understood as an effect of a pure decisiveness to confront persons who are perceived by the bullying actor as students of lower quality. In any case, due to the asymmetric character of this relation, the exercise of bullying in this case, it cannot be attributed to a return. From a normative point of view, such a behavior in the part of scientifically ambitious students in the everyday life could be characterized negatively as "arrogant" or, positively, as "quality demanding". 
These who are professionally (not scientifically) ambitious are not so strict but rather tolerant to the others, as they do not become target nor practice bullying to others. Thus, professional ambitions seem to maintain a much more "humanistic" character in contrast to the scientific ambitions rather seem to disregard the humanistic aspect. This can be attributed to the fact scientific distinction is based on the "ideal" as perceived by each student while the professional distinction is based on developing constructive relations with all people regarding them as possible future partners or clients.

Having experienced bullying by other students causes also practicing bullying as a reaction while practicing bullying leads not only to targeting, as discussed above, but also to practice conventional bullying to other students. Having practiced bullying as children is correlated with practicing bullying also as a student.

Selectiveness in friendship seems not only to protect against but also to retain the practicing of bullying, when, however, this selectiveness is based on socially constructive criteria such as politeness and friendliness. When this selectiveness is based on demanding sacrifice, such a selectiveness seems to be correlated with multidimensional bullying. This can be understood, as an effect of egoism or individualistic worldview.

In Table 6, five types are depicted: the "full victim", the "apprenticed", the "libeled scapegoat", the "ridiculed scapegoat" and the "gladiator". The "full victim" finds no support (denied to be helped) by the classmates and simultaneously he is multiply victimized by them. This type depicts a try of absolute

Table 6. Targeting for bullying (sum $=$ indegree + katz + pagerank + authority $)$.

\begin{tabular}{|c|c|c|c|c|c|}
\hline & \multicolumn{5}{|c|}{ Component } \\
\hline & $\begin{array}{c}\text { Full } \\
\text { victim }\end{array}$ & Apprenticed & $\begin{array}{c}\text { Libeled } \\
\text { scapegoat }\end{array}$ & $\begin{array}{l}\text { Ridiculed } \\
\text { scapegoat }\end{array}$ & Gladiator \\
\hline $\begin{array}{l}\text { Learn something } \\
\text { new }\end{array}$ & 0.247 & 0.809 & 0.263 & 0.030 & 0.120 \\
\hline Being exemplified & 0.170 & 0.812 & 0.172 & 0.128 & 0.016 \\
\hline Deny help & 0.403 & -0.395 & 0.442 & 0.387 & -0.003 \\
\hline Unhappiness & 0.699 & 0.111 & -0.100 & -0.240 & -0.543 \\
\hline Harassment & 0.772 & -0.055 & 0.037 & -0.442 & -0.106 \\
\hline Exclusion & 0.414 & -0.161 & 0.610 & 0.411 & -0.027 \\
\hline Spread rumor & 0.451 & -0.066 & 0.398 & -0.500 & 0.199 \\
\hline Fight & 0.495 & 0.064 & -0.311 & 0.220 & 0.665 \\
\hline $\begin{array}{c}\text { Causing } \\
\text { disagreements }\end{array}$ & 0.633 & 0.140 & -0.483 & 0.178 & -0.014 \\
\hline $\begin{array}{c}\text { Encouraging to } \\
\text { scold }\end{array}$ & 0.590 & -0.316 & -0.078 & -0.196 & 0.367 \\
\hline $\begin{array}{l}\text { Making fun at } \\
\text { one's cost }\end{array}$ & 0.593 & -0.087 & -0.264 & 0.460 & -0.312 \\
\hline
\end{tabular}

Extraction method: Principal component analysis. a 5 components extracted. 
elimination of a person as a social entity. A clearly complementary type to "full victim" is the "apprenticed". The "apprenticed" is a classmate who is inspired by others. This may be both an effect and a determinant. Namely, a student may be apprenticed to others because they respect him and thus, he is inspired by them, or he may be protected by their bullying due to his dedication to them, by which he may gain their sympathy.

The "libeled scapegoat" is a profile nested in this of "full victim". It is actually a much slighter case of victimization focused on marginalization and libeling. A similar type who is ridiculed instead of libeled is the "ridiculed scapegoat". The occurrence of such slight cases is natural, as the exclusive existence of "full victims" could lead to polarization or collapse of any social order.

Finally, the "gladiator" is a peculiar profile, as it is attacked and simultaneously encouraged to attack. This looks like a vicious circle which resembles the model of "gladiator": enduring fights but also being implicated by others in fights. He is not clearly a victim and not clearly an aggressor. Thus, he is a person appropriate for entertainment or spectacular fighting. Similar typologies concerning not bullying but verbal aggressiveness have already been suggested [47] [48] [49] [50].

In Table 7, three types are formulated: the "stimulating victimizer", the "provocateur" and the "egoist inspirator".

The first type seems to be an effect of full composition of power. He can both constructively influence others in the direction of learning and practice all forms of bullying. In normative terms, this type could be characterized as quite dangerous and challenging, because he exerts both "constructive" and "deconstructive" forms of power. This constitutes him quite invulnerable and also effective in bullying.

The "provocateur" is a profile nested in the "stimulating victimizer". Thus, it

Table 7. Practicing bullying (outdegree).

\begin{tabular}{cccc}
\hline & & Component & \\
\cline { 2 - 3 } & $\begin{array}{c}\text { Stimulating } \\
\text { victimizer }\end{array}$ & Provocateur & Egoist inspirator \\
\hline Learn something new & $\mathbf{0 . 5 2 0}$ & -0.639 & 0.293 \\
Being exemplified & $\mathbf{0 . 6 6 8}$ & -0.430 & $\mathbf{0 . 4 0 6}$ \\
Deny help & $\mathbf{0 . 5 4 0}$ & -0.062 & $\mathbf{0 . 3 8 8}$ \\
Roasting & $\mathbf{0 . 6 3 6}$ & $\mathbf{0 . 4 1 6}$ & 0.020 \\
Unhappiness & $\mathbf{0 . 7 8 1}$ & -0.043 & 0.059 \\
Harassment & $\mathbf{0 . 6 7 7}$ & 0.041 & -0.446 \\
Exclusion & $\mathbf{0 . 7 0 4}$ & -0.332 & -0.448 \\
Libeling & $\mathbf{0 . 6 9 1}$ & -0.157 & -0.469 \\
Fight & $\mathbf{0 . 5 0 2}$ & $\mathbf{0 . 4 8 3}$ & 0.155 \\
Encouraging to scold & $\mathbf{0 . 7 4 0}$ & $\mathbf{0 . 3 3 6}$ & 0.110 \\
\hline
\end{tabular}

Extraction method: Principal component analysis. a 3 components extracted. 
is not a clearly demarcated type. He consists of certain dimensions, such as roasting, fighting but also encouraging disagreements and scolding. Thus, he does not only directly cause disorder but also let others cause this. This capacity of manipulating others multiplies his deconstructiveness.

Finally, there is the type of the "egoist inspirator". On the one hand, he constitutes an example for the others but also he denies helping them. This makes his egoism more obvious and points out his tendency not to use his influence for constructing relation but rather for emphasizing his personal potential.

\section{Conclusions}

In this research, bullying has been examined as a structural phenomenon using social network analysis at students' classes and conventional statistics. Determinants and typology of bullying patterns have been detected. The following results can be regarded as the most important ones:

Female students seem to be protected against being a target of bullying while students of high semesters tend to be involved in scolding encouraged by others. Tallness, weight and mother's education level make someone a target. High grade has controversial impacts as it both makes someone a target of bullying (disagreements) but also to prevent being involved in scolding encouraged by others. Involvement in scolding in enhanced by travelling abroad for sport reasons as well as the desire of scientific or professional distinction and internet-based bullying. Selectiveness in friendship tends to prevent victimization. Thus, gender, grade and the traveling abroad for sport, the desire of distinction in science and the social selectiveness tend to protect against bullying, as they are related with persuasiveness, reputation compatibility. On the other hand, high semester, tallness, high educational influence of family and cyber-bullying appear as driving forces to certain forms of victimization, as they tend to induce jealousness, familiarization or they reveal a susceptibility to involvement.

Social and mental poorness signified by the lack of traveling abroad as well as different mentality signified by positive relations with professors seem to attract bullying. The fact that those who have experienced bullying as children still tend to experience exclusion reveals that there may be permanent attributes attracting bullying on certain persons. Moreover, being a target of libeling may even a reason for not attending lectures. Beyond targeting, female students appear to avoid practicing bullying while physical characteristics like tallness and weight as well as social parameters like education background of family and the study time prove also to be determinants of practicing bullying. High grade seems not only to protect but also to discourage someone from practicing bullying. The desire for distinction in science tends to motivate practicing bullying, though it protects against it. This asymmetry can be attributed to a pure decisiveness to promote an idea of "quality" or to protect this idea against "lower quality" people. Unlike the desire for distinction in scientific arena, the professional ambitions seem to enhance tolerance rather than bullying activity. Thus, science ambitions seem to be related with elitist ideology, unlike professional ambitions which 
seem to be related with humanism or sociability. Having experienced bullying by others causes also practicing bullying while having practiced bullying as a child is correlated with practicing bullying also as a student. Selectiveness based on politeness and friendliness (and not on egoistic demanding sacrifice) seems also to retain the practicing of bullying.

Five types of bullying targets are depicted: the "full victim" (absolute elimination of a social entity), the "apprenticed" (inspired and protected), the "libeled scapegoat" (marginalized and libeled), the "ridiculed scapegoat" (marginalized and ridiculed) and the "gladiator" (attacked and simultaneously encouraged to attack). Three types of practicing bullying are formulated: the "stimulating victimizer" (invulnerable and also effective in bullying), the "provocateur" (manipulating others multiplies his deconstructiveness) and the "egoist inspirator" (using his influence for emphasizing his personal potential and not for constructing relations).

Considering the findings mentioned above, encouraging students to pay attention to their science as well as to their professional career plan could be suggested as an effective strategy for preventing bullying. Integrating students into research could be an operational way of implementing such a strategy. In this way, teaching and research staff could strengthen the constructive socializing among students eliminating bullying. In general, the afore-mentioned determinants (driving forces) of bullying or targeting can be used as criteria for paying attention to students who need to be more integrated in such a socialization strategy and the typology could also be used as criteria for classification of students as possible targets or offenders.

Questions and challenges for future research would be to extend network sampling to other faculties and to make a comparative analysis between them (e.g. are the law and philology students as susceptible to bullying as the physical education or veterinary students?). More parameters (e.g. further detailed socio-economic or biographic elements) may also be examined as possible determinants (or effects) of bullying or targeting for bullying.

\section{References}

[1] Bekiari, A. (2012) Perceptions of Instructors' Verbal Aggressiveness and Physical Education Students' Affective Learning. Perceptual and Motor Skills, 115, 325-335. https://doi.org/10.2466/06.11.16.PMS.115.4.325-335

[2] Bekiari, A. (2014) Verbal Aggressiveness and Leadership Style of Sports Instructors and Their Relationship with Athletes' Intrisic Motivation. Creative Education, 5, 114-121. https://doi.org/10.4236/ce.2014.52018

[3] Bekiari, A. (2016) Insights into Instructors' Verbal Aggressiveness and Students' Machiavellianism through Leadership Style and Motivational Climate. European Scientific Journal, 12, 90-110. https://doi.org/10.19044/esj.2016.v12n25p90

[4] Bekiari, A., Digelidis, N. and Sakellariou, K. (2006) Perceived Verbal Aggressiveness of Coaches in Volleyball and Basketball: A Preliminary Study. Psychological Reports, 103, 526-530.

[5] Bekiari, A. and Sakellariou, K. (2003) Perceived Instructor Verbal Aggressiveness 
and Student State Learning in Physical Education. Italian Journal of Sport Sciences, 1, 251-256.

[6] Bekiari, A., Kokaridas, D. and Sakellariou, K. (2005) Verbal Aggressiveness of Physical Education Teachers and Students' Self-Reports of Behaviour. Psychological Reports, 96, 493-498. https://doi.org/10.2466/pr0.96.2.493-498

[7] Bekiari, A., Kokaridas, D. and Sakellariou, K. (2006) Associations of Students' Self-Reports of Their Teacher's Verbal Aggression, Intrinsic Motivation, and Perceptions of Reasons for Discipline in Greek Physical Education Classes. Psychological Reports, 98, 451-461. https://doi.org/10.2466/pr0.98.2.451-461

[8] Bekiari, A. and Manoli, P. (2016) EFL Teacher Verbal Aggressiveness and Argumentativeness and Student Socio-Affective Strategy Use and Affective Learning: Exploring Possible Associations. Journal of Teacher Education and Educators, 5, 154-171.

[9] Bekiari, A., Patsiaouras, A., Kokaridas, D. and Sakellariou, K. (2006) Verbal Aggressiveness and State Anxiety of Volleyball Players and Coaches. Psychological Reports, 99, 630-634.

[10] Bekiari, A., Perkos, S. and Gerodimos, V. (2015) Verbal Aggression in Basketball: Perceived Coach Use and Athlete Intrinsic and Extrinsic Motivation. Journal of Physical Education and Sport, 15, 96-102.

[11] Bekiari, A. and Pylarinou, M. (2016) Instructor Argumentativeness and Socio-Communicative Style and Student Discipline: Using Physical Education Students' Class as an Illustration. Open Journal of Social Sciences, 5, 122-136. https://doi.org/10.4236/jss.2017.53011

[12] Bekiari, A. and Syrmpas, I. (2015) Coaches' Verbal Aggressiveness and Motivational Climate as Predictors of Athletes' Satisfaction. British Journal of Education, Society and Behavioural Science, 9, 318-329. https://doi.org/10.9734/BJESBS/2015/17757

[13] Hassandra, M., Bekiari, A. and Sakellariou, K. (2007) Physical Education Teacher's Verbal Aggression and Student's Fair Play Behaviors. The Physical Educator, 64, 94-101.

[14] Manoli, P. and Bekiari, A. (2015) EFL Teachers' Verbal Aggressiveness and Students' Intrinsic Motivation and Social-Affective Strategy Use: Investigating Possible Relations. Advances in Research, 5, 1-13. https://doi.org/10.9734/AIR/2015/19692

[15] Syrmpas, I. and Bekiari, A. (2015) The Relationship between Perceived Physical Education Teacher's Verbal Aggressiveness and Argumentativeness with Students' Interpersonal Attraction. Inquiries in Sport and Physical Education, 13, 21-32.

[16] Olweus, D. (1973b) Personality and Aggression. In: Cole, J.K. and Jensen, D.D., Eds., Nebrasca Symposium on Motivation, University of Nebrasca Press, Lincoln.

[17] Rivers, I. and Smith, P.K. (1994) Types of Bullying Behaviour and Their Correlates. Aggressive Behavior, 20, 359-368. https://doi.org/10.1002/1098-2337(1994)20:5<359::AID-AB2480200503>3.0.CO;2-J

[18] Cassidy, T. (2008) Bullying and Victimization in School Children: The Role of Social Identity, Problem-Solving Style, and Family and School Context. Social Psychology of Education, 12, 63-76. https://doi.org/10.1007/s11218-008-9066-y

[19] Juvonen, J., Nishina, A. and Graham, S. (2001) Self-Views versus Peer Perception of Victim Status among Early Adolescents. In: Juvonen, J. and Graham, S., Eds., Peer Harassment in School: The Plight of the Vulnerable and Victimized, The Guilford Press, New York, 105-124.

[20] Chaux, E., Molano, A. and Podlesky, P. (2009) Socio-Economic, Socio-Political and Socio-Emotional Variables Explaining School Bullying: A Country-Wide Multilevel 
Analysis. Aggressive Behavior, 35, 520-529. https://doi.org/10.1002/ab.20320

[21] Menesini, E., Modena, M. and Tani, F. (2009) Bullying and Victimization in Adolescence: Concurrent and Stable Roles and Psychological Health Symptoms. The Journal of Genetic Psychology, 170, 115-134. https://doi.org/10.3200/GNTP.170.2.115-134

[22] Coyne, S.M., Archer, J. and Eslea, M. (2006) "We're Not Friends Anymore! Unless...": The Frequency and Harmfulness of Indirect, Relational, and Social Aggression. Aggressive Behavior, 32, 294-307. https://doi.org/10.1002/ab.20126

[23] Garner, P. and Hinton, T. (2010) Emotional Display Rules and Emotion Self-Regulation: Associations with Bullying and Victimization in Community-Based after School Programs. Journal of Community and Applied Social Psychology, 20, 480 496. https://doi.org/10.1002/casp.1057

[24] Jantzer, A.M., Hoover, J.H. and Narloch, R. (2006) The Relationship between School-Aged Bullying and Trust, Shyness and Quality of Friendships in Young Adulthood: A Preliminary Research Note. School Psychology International, 27, 146 156. https://doi.org/10.1177/0143034306064546

[25] Chapell, M., Casey, D., De la Cruz, C., Ferrell, J., Forman, J., Lipkin, R. and Whittaker, S. (2004) Bullying in College by Students and Teachers. Adolescence, 39, $53-$ 64.

[26] Adams, F.D. and Lawrence, G.J. (2011) Bullying Victims: The Effects Last into College. American Secondary Education, 40, 4-13.

[27] Hawker, D.S.J. and Boulton, M.J. (2000) Twenty Years' Research on Peer Victimization and Psychosocial Maladjustment: A Meta-Analytic Review of Cross-Sectional Studies. Journal of Child Psychology and Psychiatry and Allied Disciplines, 41, 441-455. https://doi.org/10.1111/1469-7610.00629

[28] Goodboy, A.K., Martin, M.M. and Goldman, Z.W. (2016) Students' Experiences of Bullying in High School and Their Adjustment and Motivation during the First Semester of College. Western Journal of Communication, 80, 60-78. https://doi.org/10.1080/10570314.2015.1078494

[29] Olweus, D. (1994) Bullying at School: Long-Term Outcomes for the Victims and an Effective School-Based Intervention Program. In: Huesmann, L.R., Ed., Aggressive Behavior: Current Perspectives, Plenum Press, New York, 97-130. https://doi.org/10.1007/978-1-4757-9116-7_5

[30] Ttofi, M., Farrington, D., Lösel, F. and Loeber, R. (2011) Do the Victims of School Bullies Tend to Become Depressed Later in Life? A Systematic Review and MetaAnalysis of Longitudinal Studies. Journal of Aggression, Conflict and Peace Research, 3, 63-73. https://doi.org/10.1108/17596591111132873

[31] Suresh, S. and Tipandjan, A. (2012) School Bullying Victimization and College Adjustment. Journal of the Indian Academy of Applied Psychology, 38, 68-73.

[32] Bekiari, A., Deliligka, S. and Hasanagas, N. (2017) Analyzing Networks of Verbal Aggressiveness and Motivation. Psychology, 8, 495-515.

https://doi.org/10.4236/psych.2017.83031

[33] Bekiari, A. and Hasanagas, N. (2015) Verbal Aggressiveness Exploration through Complete Social Network Analysis: Using Physical Education Students' Class as an Illustration. International Journal of Social Science Studies, 3, 30-49. https://doi.org/10.11114/ijsss.v3i3.729

[34] Bekiari, A. and Hasanagas, N. (2016) Sociological Insights in the Education System: "Unlocking" the Power Relations. Afoi Kyriakidi Editions S.A., Thessaloniki.

[35] Bekiari, A. and Hasanagas, N. (2016) "Educating" in Physical Education. Theoreti- 
cal Approaches and Practical Inquiries. Afoi Kyriakidi Editions S.A., Thessaloniki.

[36] Bekiari, A. and Hasanagas, N. (2016) Suggesting Indicators of Superficiality and Purity in Verbal Aggressiveness. An Application in Adult Education Class Networks of Prisoners. Open Journal of Social Sciences, 4, 279-292. https://doi.org/10.4236/jss.2016.43035

[37] Theoharis, D., Bekiari, A. and Koustelios, A. (2017) Exploration of Determinants of Verbal Aggressiveness and Leadership through Network Analysis and Conventional Statistics. Using School Class as an Illustration. Sociology Mind, 7, 27-43. https://doi.org/10.4236/sm.2017.72003

[38] Bekiari, A., Deliligka, S. and Koustelios, A. (2016) Examining Relations of Aggressive Communication in Social Networks. Social Networking, 6, 38. https://doi.org/10.4236/sn.2017.61003

[39] Bekiari, A., Hasanagas, N., Theoharis, D., Kefalas, I. and Vasilou, A. (2015) The Role of Mathematical Object and the Educational Environment to Students' Interpersonal Relationships: An Application of Full Social Network Analysis. Proceedings of the 32 nd Congress Greek Mathematical Society (with International Participation), Kastoria, 799-812.

[40] Bekiari, A. and Spyropoulou, S. (2016) Exploration of Verbal Aggressiveness and Interpersonal Attraction through Social Network Analysis: Using University Physical Education Class as an Illustration. Open Journal of Social Sciences, 4, 145-155. https://doi.org/10.4236/jss.2016.46016

[41] Hasanagas, N. and Bekiari, A. (2015) Depicting Determinants and Effects of Intimacy and Verbal Aggressiveness Target through Social Network Analysis. Sociology Mind, 5, 162-175. https://doi.org/10.4236/sm.2015.53015

[42] Hasanagas, N. and Bekiari, A. (2017) An Exploration of the Relation between Hunting and Aggressiveness: Using Inmates Networks at Prison Secondary School as an Illustration. Social Networking, 6, 19-37. https://doi.org/10.4236/sn.2017.61002

[43] Bekiari, A. and Digelidis, N. (2015) Measuring Verbal Aggressiveness in Sport and Education. International Journal of Physical Education, 52, 12-21.

[44] Theoharis, D. and Bekiari, A. (2017) Cumulative Hierarchy Analysis (Katz Centrality) on Leadership Networks of Learning Communities. Proceedings of the 9 th International Congress Mathematical Society, Thessaloniki, 17-19 March 2017, 285297.

[45] Theoharis, D. and Bekiari, A. (2016) The Influence of Mathematics and Learning Environment in Verbal Aggressiveness and Interpersonal Relations: A Dynamic Analysis of Social Networks. Proceedings of the 8 th International Congress Mathematical Society, Thessaloniki, 30 March-3 April 2016, 415-428.

[46] Theoharis, D. and Bekiari, A. (2016) Social Networks Analysis of Centrality: Case Study in Leadership Networks. Proceedings of the 33nd Congress Greek Mathematical Society (with International Participation), Chania, 4-6 November 2016, 250 260.

[47] Bekiari, A., Nikolaidou, Z. and Hasanagas, N. (2017) Typology of Motivation and Aggression on the Basis of Social Network Variables: Examples of Complementary and Nested Behavioral Types through Conventional Statistics. Social Networking, 6, 135-147. https://doi.org/10.4236/sn.2017.62008

[48] Bekiari, A. and Tsiana, I. (2016) Exploring Instructors' Verbal Aggressiveness and Students' Personal Orientations and Reasons of Discipline in Physical Education Class. Advances in Physical Education, 6, 158-168.

https://doi.org/10.4236/ape.2016.63018 
[49] Bekiari, A. and Petanidis, D. (2016) Exploring Teachers' Verbal Aggressiveness through Interpersonal Attraction and Students' Intrinsic Motivation. Open Journal of Social Sciences, 4, 72-85. https://doi.org/10.4236/jss.2016.412007

[50] Bekiari, A. and Tsaggopoulou, T. (2016) Verbal Aggressiveness and Affective Learning in Physical Education. Advances in Physical Education, 6, 406-418. https://doi.org/10.4236/ape.2016.64041

\section{Scientific Research Publishing}

Submit or recommend next manuscript to SCIRP and we will provide best service for you:

Accepting pre-submission inquiries through Email, Facebook, LinkedIn, Twitter, etc. A wide selection of journals (inclusive of 9 subjects, more than 200 journals)

Providing 24-hour high-quality service

User-friendly online submission system

Fair and swift peer-review system

Efficient typesetting and proofreading procedure

Display of the result of downloads and visits, as well as the number of cited articles Maximum dissemination of your research work

Submit your manuscript at: http://papersubmission.scirp.org/

Or contact jcc@scirp.org 